\title{
Search for a high mass diphoton resonance using the ATLAS detector
}

\author{
Faïrouz Malek on behalf of the ATLAS Collaboration ${ }^{1, a}$ \\ ${ }^{1}$ LPSC-Grenoble, CNRS-IN2P3, Université Grenoble-Alpes
}

\begin{abstract}
High-mass states decaying into two photons are predicted in many extensions of the Standard Model (SM). The diphoton final state provides a clean experimental signature with good invariant mass resolution and moderate backgrounds. Searches for highmass resonances decaying into two photons for a spin- 0 or spin- 2 state are presented. The latest ATLAS results using p-p collision data at $13 \mathrm{TeV}$ and covering a large mass range are discussed.
\end{abstract}

\section{Introduction}

Searches for high-mass resonances decaying into two photons using CERN Large Hadron Collider (LHC) proton-proton collision data at $\sqrt{s}=13 \mathrm{TeV}$ recorded in 2015 by the ATLAS detector and corresponding to an integrated luminosity of $3.2 \mathrm{fb}^{-1}$ are described.

During the LHC Run-1 period, at $\sqrt{s}=7 \mathrm{TeV}$ and $8 \mathrm{TeV}$, more than $20 \mathrm{fb}^{-1}$ of data were recorded and searches for diphoton resonances have been reported by the ATLAS and CMS collaborations [15]. The analyses using the 2015 LHC pp data have also been published [6, 7].

The analyses were performed using two benchmark models: the diphoton decay resulting from a hypothetical particle of spin-0 or spin- 2 . This implies that diphotons have different kinematic properties depending on the model used. This means that one needs to apply two different selections, with looser kinematic selection requirements for the spin-2 resonance search. The photon identification criteria and the event pre-selection are common to both searches.

\section{Search for a $\gamma \gamma$ resonance}

\subsection{The spin-2 case}

The search for a spin-2 $\gamma \gamma$ resonance uses the Randall-Sundrum (RS) model [8] graviton as a benchmark. In this model, we look for the lightest Kaluza-Klein [9] spin-2 graviton excitation $\left(G^{*}\right)$ with a dimensionless coupling $k / \bar{M}_{\mathrm{Pl}}$, where $\bar{M}_{\mathrm{Pl}}=M_{\mathrm{Pl}} / \sqrt{8 \pi}$ is the reduced Planck scale and $k$ the curvature scale of the extra dimension. The lightest graviton excitation is expected to be a fairly narrow resonance for $k / \bar{M}_{\mathrm{Pl}}<0.3$ [10], with the width given by $1.44\left(k / \bar{M}_{\mathrm{Pl}}\right)^{2} m_{G^{*}}$, where $m_{G^{*}}$ is the mass of the lightest graviton state. For $k / \bar{M}_{\mathrm{Pl}}=0.1$, the natural width increases from $11 \mathrm{GeV}$ at $m_{G^{*}}=800 \mathrm{GeV}$

ae-mail: fmalek@1psc.in2p3.fr 
to $30 \mathrm{GeV}$ at $m_{G^{*}}=2200 \mathrm{GeV}$. The shape of the invariant mass distribution of the main background from the production of prompt photon pairs is estimated from theoretical computations, and the contribution from the reducible background of events where at least one jet is misidentified as a photon is added from data-driven estimates. The search is performed in the mass range above $500 \mathrm{GeV}$ and in the $k / \bar{M}_{\mathrm{Pl}}$ range 0.01 to 0.3 , searching for an excess over the estimated background diphoton invariant mass distribution. To model such an excess, the RS graviton resonance shape is convolved with the experimental resolution.

\subsection{The spin-0 case}

Spin-0 $\gamma \gamma$ resonances are predicted in theories with an extended Higgs sector [11-17]. The search for a spin-0 resonance uses a more restricted kinematic range for the photon selection. The background is estimated by fitting the diphoton invariant mass distribution to an analytical function, searching for an excess modelled by a spin- 0 resonance convolved with the experimental resolution. The search is performed in the mass range $200-2000 \mathrm{GeV}$, and for width values up to $10 \%$ of the mass of the hypothesized particle.

\section{ATLAS detector}

The ATLAS detector [18] is a multi-purpose detector with a forward-backward symmetric cylindrical geometry. ${ }^{1}$ At small radii, the inner detector (ID) is immersed in a $2 \mathrm{~T}$ magnetic field and is made up of fine-granularity pixel and microstrip detectors covering a pseudorapidity range $|\eta|<2.5$. A gas-filled straw-tube transition radiation tracker (TRT) complements the silicon tracker at larger radii and also provides electron identification capabilities based on transition radiation. The electromagnetic (EM) calorimeter is a lead/liquid-argon sampling calorimeter with accordion geometry. The calorimeter is divided into a barrel section covering $|\eta|<1.475$ and two end-cap sections covering $1.375<|\eta|<3.2$. Hadronic calorimetry in the region $|\eta|<1.7$ uses steel absorbers and scintillator tiles as the active medium. Liquid-argon calorimetry with copper absorbers is used in the hadronic end-cap calorimeters, which cover the region $1.5<|\eta|<3.2$. A forward calorimeter using copper or tungsten absorbers with liquid argon completes the calorimeter coverage up to $|\eta|=4.9$. The muon spectrometer measures the deflection of muon tracks within $|\eta|<2.7$. The deflection is provided by a toroidal magnetic field from air-core superconducting magnets, with an integral of approximately $3 \mathrm{~T} \cdot \mathrm{m}$ and $6 \mathrm{~T} \cdot \mathrm{m}$ in the central and end-cap regions, respectively. The muon spectrometer is instrumented with trigger chambers covering $|\eta|<2.4$. Events are selected using a first-level trigger implemented in custom electronics, which reduces the event rate to a design value of $100 \mathrm{kHz}$. Software algorithms with access to the full detector information are then used in the high-level trigger to yield a recorded event rate of about $1 \mathrm{kHz}[19]$.

\section{Photon selection}

Photon and electron candidates are reconstructed from clusters of energy deposited in the electromagnetic calorimeter. Candidates without a matching track or reconstructed conversion vertex in the inner detector are classified as unconverted photons. Those with a matching reconstructed conversion

\footnotetext{
${ }^{1}$ The ATLAS experiment uses a right-handed coordinate system with its origin at the nominal interaction point (IP) in the centre of the detector and the $z$-axis along the beam pipe. The $x$-axis points from the IP to the centre of the LHC ring, and the $y$-axis points upward. Cylindrical coordinates $(r, \phi)$ are used in the transverse plane, $\phi$ being the azimuthal angle around the beam pipe. The pseudorapidity is defined in terms of the polar angle $\theta$ as $\eta=-\ln \tan (\theta / 2)$.
} 
vertex or a matching track, consistent with originating from a photon conversion, are classified as converted photons. Those matched to a track consistent with originating from an electron produced in the beam interaction region are kept as electrons.

Only photon candidates with $|\eta|<2.37$ are considered, not including the transition region $1.37<$ $|\eta|<1.52$ between the barrel and end-cap calorimeters.

The efficiency of the photon identification increases with the transverse energy of the photon $E_{\mathrm{T}}$ from $85 \%$ at $50 \mathrm{GeV}$ to $95 \%$ at $200 \mathrm{GeV}$. For $E_{\mathrm{T}}>50 \mathrm{GeV}$, the uncertainty in the photon identification efficiency varies between $\pm 1 \%$ and $\pm 5 \%$ depending on $\eta$ and $E_{\mathrm{T}}$ [20].

To further reject the background from jets misidentified as photons, the photon candidates are required to be isolated using both calorimeter and tracking detector information. The calorimeter isolation variable, $E_{\mathrm{T}}^{\text {iso }}$, is defined as the sum of the $E_{\mathrm{T}}$ of energy clusters deposited in a cone of size $\Delta R=\sqrt{(\Delta \eta)^{2}+(\Delta \phi)^{2}}=0.4$ around the photon candidate, excluding an area of size $\Delta \eta \times \Delta \phi=$ $0.125 \times 0.175$ centred on the photon cluster; the expected photon energy deposit outside the excluded area is subtracted. The pile-up and underlying-event contribution to the calorimeter isolation variable is subtracted from the isolation energy event-by-event [21-23]. The selection requirement on the calorimeter isolation variable is defined by $E_{\mathrm{T}}^{\text {iso }}<0.022 E_{\mathrm{T}}+2.45 \mathrm{GeV}$. The track isolation variable $\left(p_{\mathrm{T}}^{\text {iso }}\right)$ is defined as the scalar sum of the transverse momenta of the tracks in a cone of $\Delta R=0.2$ around the photon candidate. The tracks are required to have $p_{\mathrm{T}}>1.0 \mathrm{GeV}$ and to be consistent with originating from the diphoton primary vertex. For converted photons, the one or two tracks associated with the photon conversion are excluded from the $p_{\mathrm{T}}^{\text {iso }}$ computation. The requirement applied for the track isolation variable is $p_{\mathrm{T}}^{\text {iso }}<0.05 E_{\mathrm{T}}$.

\section{Data and simulated event samples}

Data were collected by the ATLAS detector in 2015 using $p p$ collisions at a centre-of-mass energy of $\sqrt{s}=13 \mathrm{TeV}$. Events were recorded using a diphoton trigger with $E_{\mathrm{T}}$ thresholds of $35 \mathrm{GeV}$ and $25 \mathrm{GeV}$ for the leading and subleading photon candidates, respectively. The trigger has a signal efficiency close to $99 \%$ for events fulfilling the final event selections. After data-quality requirements, the data sample corresponds to an integrated luminosity of $3.2 \mathrm{fb}^{-1}$. The measurement of the integrated luminosity has an uncertainty of $\pm 5 \%$ [24].

Simulated Monte Carlo (MC) samples are used to optimize the search strategy and to study background sources. The generated events are passed through a full detector simulation [25] based on GEant4 [26]. Pile-up from additional $p p$ collisions in the same and neighbouring bunch crossings is simulated by overlaying each MC event with a variable number of simulated inelastic $p p$ collisions generated using PyтнIA8 with the A2 tune [27]. The MC events are weighted to reproduce the distribution of the average number of interactions per bunch crossing observed in the data.

\subsection{Spin-2 signal}

Signal samples for the RS graviton model are generated using Pythia8 [28], version 8.186, with the NNPDF23LO [29] parton distribution functions (PDF) and the A14 [30] set of tuned parameters for the underlying event, for different choices of the graviton mass and the parameter $k / \bar{M}_{\mathrm{Pl}}$. A mass range from $500 \mathrm{GeV}$ to $5000 \mathrm{GeV}$ and $k / \bar{M}_{\mathrm{Pl}}$ values from 0.01 to 0.3 are chosen. Samples for any mass or $k / \bar{M}_{\mathrm{Pl}}$ value are obtained by reweighting an event sample generated with a uniform mass distribution using the Breit-Wigner and parton luminosity terms. 


\subsection{Spin-0 signal}

The signal in the spin-0 particle search is simulated as if it were a SM Higgs boson produced in $p p$ collisions via gluon fusion and decaying into two photons. MC samples are produced for different hypotheses of the spin- 0 boson mass $\left(m_{X}\right)$ in the range $200 \mathrm{GeV}$ to $2000 \mathrm{GeV}$ and of the decay width $\left(\Gamma_{X}\right)$ up to $10 \%$ of $m_{X}$. Gluon fusion events are generated with PowHEG-Box [31,32], version 2, interfaced with Pyтніa8 for the underlying event, parton showering and hadronization. To model signals with large decay widths, a function parameterizing the theoretical line-shape of the resonance is used. The PowHEG-Box implementation of a large-width spin-0 resonance with couplings like those of the Higgs boson in the SM is chosen for this function.

\subsection{Background}

Events containing two prompt photons, representing the largest irreducible background to the search, are simulated using the SHERPA [33] generator version 2.1.1. Matrix elements are calculated with up to two partons at leading order in QCD and merged with the SHERPA parton shower [34] using the ME+PS@LO prescription [35]. Samples of the photon+jet reducible background component are also generated using SHERPA.

\section{Event selection}

For the spin-2 resonance, the transverse energy of each photon is required to satisfy $E_{\mathrm{T}}>55 \mathrm{GeV}$. For the spin-0 resonance, we apply tighter selections which were optimized on simulated background and signal samples. For a given value of $m_{\gamma \gamma}$, the transverse energy is required to be $E_{\mathrm{T}}>0.4 m_{\gamma \gamma}$ for the photon with the highest $E_{\mathrm{T}}$ and $E_{\mathrm{T}}>0.3 m_{\gamma \gamma}$ for the photon with the second-highest $E_{\mathrm{T}}$.

The selected samples consist of events from diphoton production, followed by photon+jet production, with one jet misidentified as a photon, and dijet production with two jets misidentified as photons. Background sources from Drell-Yan, $W \gamma$ or $Z \gamma$ production, with either one or two isolated electrons misidentified as photons, are negligible.

Control regions are built from events failing the isolation requirement and/or some of the tight photon identification requirements, Ref. [36, 37]. They are used to estimate the relative contribution of the various sources of background directly from data. Figure 1 shows the decomposition of the selected data sample into the contributions from diphoton, photon+jet or jet+photon, and dijet events for both selections and the corresponding purities, defined as the ratio of diphoton events to the total number of events in the sample. The purity is $\left(94_{-7}^{+3}\right) \%$ for the spin- 2 selection and $\left(93_{-8}^{+3}\right) \%$ for the spin- 0 selection. The estimate of the uncertainties is sensitive to the small number of events in some of the control regions.

For the spin-2 resonance search, the acceptance for the benchmark RS graviton model varies from $66 \%$ at a mass of $500 \mathrm{GeV}$, to $91 \%$ at a mass of $5000 \mathrm{GeV}$. For the spin-0 resonance search, the acceptance ranges from $52 \%$ to $62 \%$ in the mass range from $200 \mathrm{GeV}$ to $700 \mathrm{GeV}$ for a particle similar to a Higgs boson produced by gluon fusion and is almost constant above $700 \mathrm{GeV}$.

\section{Signal modelling}

The invariant mass distribution of the diphoton pair for the signal is expected to peak near the assumed mass of the new resonance, with a spread given by the convolution of its intrinsic decay width with the experimental resolution. For both searches, the experimental resolution of the invariant mass is 

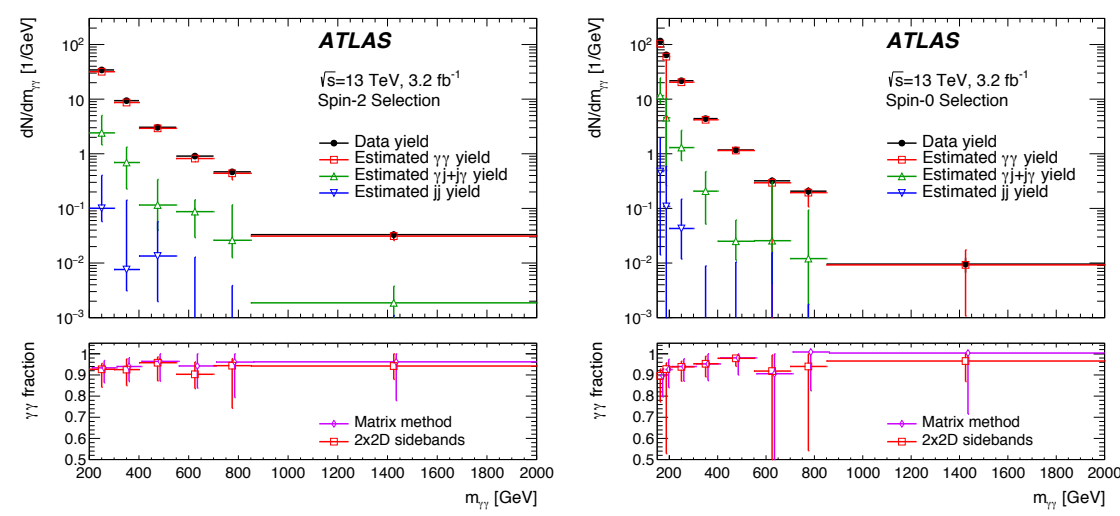

Figure 1. The diphoton invariant mass distributions (upper panels) of the data for the spin-2 and spin- 0 selections and their decomposition into contributions from genuine diphoton, photon + jet plus jet+photon and jet + jet events. The bottom panels show the purity of diphoton events [6]. The total uncertainties are shown, including statistical and systematic components.

modelled with a double-sided Crystal Ball (DSCB) function. Interference effects between signal and background are ignored.

For the spin-2 resonance search, the signal mass distribution for any value of the mass and $k / \bar{M}_{\mathrm{Pl}}$ is obtained by a convolution of the intrinsic detector resolution, modelled by a DSCB function, with the predicted distribution of the mass line-shape at generator level.

For the spin-0 resonances with larger natural widths, the reconstructed line-shapes are well described by DSCB functions. The function effectively parameterizes the combined effects of the theoretical line-shape and the detector response for a signal with width values up to $10 \%$ of the resonance mass.

\section{Background estimates}

Two different methods are used to estimate the background contributions to the $m_{\gamma \gamma}$ distribution: MC extrapolation method and functional-form approach. In the spin-2 search, the shape of the invariant mass distribution of the main diphoton background is predicted using the next-to-leading (NLO) order in QCD DIPHox [38] computation, version 1.3.2. The background from photon+jet and dijet production is added using control samples from the data. The second approach, more appropriate for the spin-0 search, is based on using a smooth functional form, with fully data-driven parameters to model the total background. In this approach, the mass distribution from data is fitted in the range above $150 \mathrm{GeV}$.

The two methods used for the background estimate were compared in the mass range where they are both used (500-5000 GeV for spin-2 and 200-2000 GeV for spin-0). For the MC extrapolation method, the statistical uncertainty is directly related to the total number of events in the data sample and thus is at the level of $\pm 1.5 \%$ for the spin-2 resonance search selection. For the functional-form approach, the statistical uncertainty originating from the uncertainty in the determination of the parameters of the function from the fit to the data is larger, especially at high masses while the systematic uncertainty is given directly by the "spurious" signal uncertainty, which depends on the signal 
hypothesis being considered. This systematic uncertainty is lower than that of the MC extrapolation approach. For all masses, the total uncertainty in the background estimate is significantly smaller than the expected statistical fluctuations of the background yield in the different signal regions being considered. There is good agreement between the background predictions from the two methods.

\section{Results}

The diphoton mass distributions from the two event samples are fitted assuming the background-only or signal-plus-background hypotheses. The uncertainties related to the overall normalization of the signal yield only affect the limits on the production cross sections, while the uncertainty in the signal mass resolution affects both the compatibility with the background-only hypothesis and the limits on the production cross section. The signal mass resolution uncertainty ranges, depending on the mass, from 20 to $60 \%$ for spin- 2 and from 30 to $60 \%$ for spin- 0 . The uncertainties in the background estimates affect both the compatibility with the background-only hypothesis and the cross-section limits. The relative systematic uncertainty in the background after the fit to the data is typically $\pm 2 \%$ to $\pm 4 \%$ at masses near $750 \mathrm{GeV}$ depending on the selection and on the method used to compute the background.
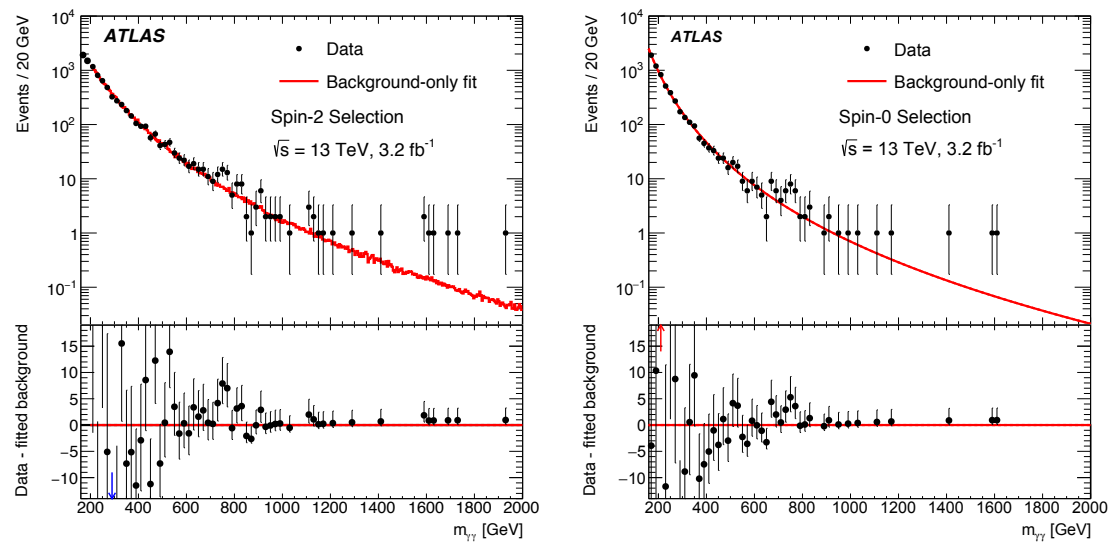

Figure 2. Distribution of the diphoton invariant mass for the selection used in the search for a spin-2 and a spin-0 resonance, with the best background-only fit [6]. The difference between the data and the fit is shown in the bottom panel for $m_{y \gamma}>200 \mathrm{GeV}$. The arrow shown in the lower panel indicates a values outside the range with more than one standard deviation. There is no data event with $m_{\gamma \gamma}>2000 \mathrm{GeV}$.

Figure 2 shows the diphoton invariant mass distributions for the events selected in the spin-2 (spin0) resonance search together with the best background-only fit using the MC extrapolation approach (the functional-form approach). For the spin-2 case, the largest deviation from the background-only hypothesis is observed near a mass of $750 \mathrm{GeV}$, for a $k / \bar{M}_{\mathrm{Pl}}$ value of 0.23 , corresponding to a local excess of 3.8 standard deviations. The width associated with $k / \bar{M}_{\mathrm{Pl}}=0.23$ at $m_{G^{*}}=750 \mathrm{GeV}$ is $57 \mathrm{GeV}$. The global significance evaluated using the search region of 500-2000 GeV in mass and $0.01-0.3$ in $k / \bar{M}_{\mathrm{Pl}}$ is 2.1 standard deviations. For the spin-0 resonance search, the largest deviation is also observed near a mass of $750 \mathrm{GeV}$. It corresponds to a local excess over the backgroundonly hypothesis with a significance of 3.9 standard deviations for a width of $45 \mathrm{GeV}$. The global 
significance evaluated using the search region of $200-2000 \mathrm{GeV}$ in mass and $0 \%-10 \%$ in $\Gamma_{X} / m_{X}$ is 2.1 standard deviations.

The compatibility of the excesses observed in the two analyses is assessed with a bootstrap statistical procedure, assuming a common signal. If the spin-2 (spin-0) signal is assumed, the two analyses are compatible within $0.5(0.2)$ standard deviations.

\section{Cross-section limits}

Since for the spin-2 case a larger model dependence exists and the analysis is performed for a specific benchmark model of a spin-2 graviton, limits on the total cross section times branching ratio to two photons are given assuming specific model parameters. Figure 3 is shown for a $k / \bar{M}_{\mathrm{Pl}}$ value of 0.1. The predicted cross sections are computed at LO in QCD using PyTHIA8. The uncertainty band represents the PDF uncertainty estimated from the variations of the NNPDF23LO PDF set. Outside of the excess region, the observed limit on the cross section times branching ratio is of $1 \mathrm{fb}$ for an RS graviton mass between $500 \mathrm{GeV}$ and $5000 \mathrm{GeV}$ for the $k / \bar{M}_{\mathrm{Pl}}=0.1$ and the graviton mass is excluded to $3.2 \mathrm{TeV}$, a neat improvement from the $8 \mathrm{TeV}$ reults $(2.66 \mathrm{TeV})$.

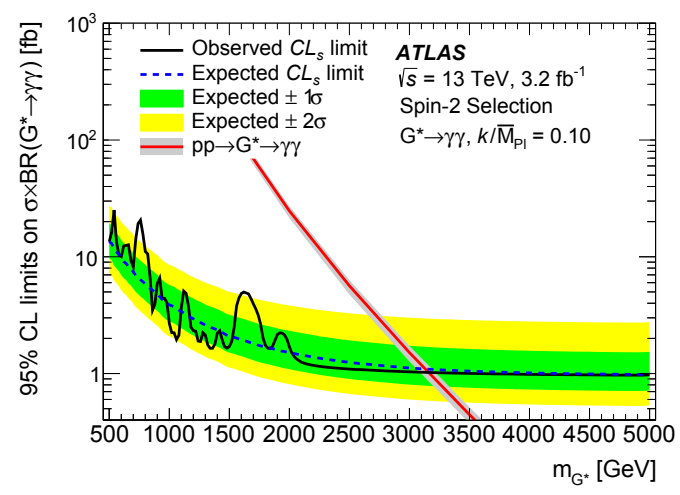

Figure 3. Upper limits on the production cross section of an RS graviton as a function of the assumed mass, for a $k / \bar{M}_{\mathrm{Pl}}$ value of 0.1 [6]. The predicted cross section times branching ratio to two photons for the RS graviton model, computed at LO, is also shown. The uncertainty in the cross-section values represent the PDF uncertainty.

For the spin-0 case, limits are interpreted in a nearly model-independent way in terms of the fiducial cross section, defined as the product of the cross section times the branching ratio to two photons within the fiducial acceptance defined. Figure 4 shows the limits on the signal fiducial cross section times branching ratio to two photons as a function of the hypothesized mass for various assumptions about the width. Except near $750 \mathrm{GeV}$, the observed limit is in agreement with the expected limit assuming the background-only hypothesis.

\section{Conclusion}

Search for high mass resonances decaying into two photons in the ATLAS experiment at the LHC are presented. The analyses are performed with $p p$ collision data corresponding to an integrated luminosity of $3.2 \mathrm{fb}^{-1}$ at a centre-of-mass energy of $\sqrt{s}=13 \mathrm{TeV}$, recorded in 2015 . The analyses 

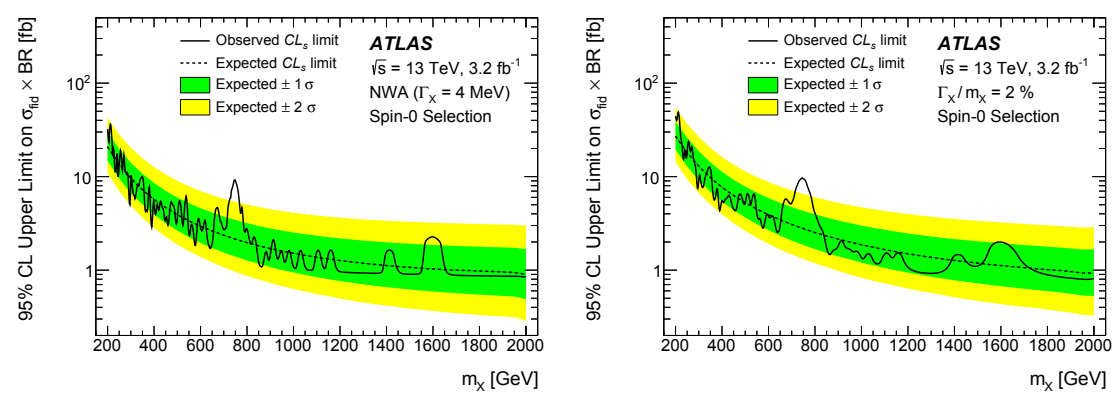

Figure 4. Upper limits on the fiducial cross section at $\sqrt{s}=13 \mathrm{TeV}$ of a spin-0 particle as a function of the assumed mass $m_{X}$, for different values of the decay width divided by the mass [6]. In (Left) picture a narrowwidth signal, with $\Gamma=4 \mathrm{MeV}$, is assumed.

were optimized for two benchmark searches: spin-2 Randall-Sundrum graviton resonance with mass above $500 \mathrm{GeV}$ and spin-0 resonance with mass above $200 \mathrm{GeV}$. The data are consistent with the background-only hypothesis and 95\% CL limits are derived on the cross section for the production of the two benchmark resonances as a function of their masses and widths. The largest deviation from the background-only hypothesis is observed in a broad region near a mass of $750 \mathrm{GeV}$ and with a width of about $50 \mathrm{GeV}$, with global significances of 2.1 standard deviations for both searches.

The intriguing resonance at $750 \mathrm{GeV}$ decaying into photon pairs, which caused considerable interest from the 2015 data, has not reappeared in the much larger 2016 data set and thus appears to be a statistical fluctuation as shown in 2016 summer conferences [39]. 


\section{References}

[1] ATLAS Collaboration, New J. Phys. 15 (2013) 043007.

[2] CMS Collaboration, Phys. Rev. Lett. 108 (2012) 111801.

[3] ATLAS Collaboration, Phys. Rev. D 92 (2015) 032004.

[4] ATLAS Collaboration, Phys. Rev. Lett. 113 (2014) 171801.

[5] CMS Collaboration, Phys. Lett. B 750 (2015) 494.

[6] ATLAS Collaboration, JHEP 09 (2016) 001.

[7] CMS Collaboration, Phys. Rev. Lett. 117 (2016) 051802.

[8] L. Randall and R. Sundrum, Phys. Rev. Lett. 83 (1999) 3370.

[9] T. Appelquist, A. Chodos and P. Freund, Modern Kaluza-Klein theories, vol. 65, Frontiers in Physics, Addison-Wesley, 1987.

[10] H. Davoudiasl, J. Hewett and T. Rizzo, Phys. Rev. Lett. 84 (2000) 2080.

[11] A. Hill and J.J. van der Bij, Phys. Rev. D 36 (1987) 3463.

[12] M. J. G. Veltman and F. J. Yndurain, Nucl. Phys. B 325 (1989) 1.

[13] T. Binoth and J.J. van der Bij, Z. Physics. C 75 (1997) 17.

[14] R. Schabinger and J. D. Wells, Phys. Rev. D 72 (2005) 093007.

[15] B. Patt and F. Wilczek, arXiv: hep-ph/0605188.

[16] G. M. Pruna and T. Robens, Phys. Rev. D 88 (2013) 115012.

[17] T. D. Lee, Phys. Rev. D 8 (1973) 1226.

[18] ATLAS Collaboration, JINST 3 (2008) S08003.

[19] ATLAS Collaboration, ATL-DAQ-PUB-2016-001, url: http://cds.cern.ch/record/2136007.

[20] ATLAS Collaboration, arXiv: 1606.01813 [hep-ex].

[21] ATLAS Collaboration, Phys. Rev. D 83 (2011) 052005.

[22] M. Cacciari, G. P. Salam and G. Soyez, JHEP 04 (2008) 005.

[23] M. Cacciari, G. P. Salam and S. Sapeta, JHEP 10 (2010) 065.

[24] ATLAS Collaboration, Eur. Phys. J. C 73 (2013) 2518.

[25] ATLAS Collaboration, Eur. Phys. J. C 70 (2010) 823.

[26] S. Agostinelli et al., Nucl. Instrum. Meth. A 506 (2003) 250.

[27] ATLAS Collaboration, ATL-PHYS-PUB-2012-003, url: http://cds.cern.ch/record/1474107.

[28] T. Sjöstrand, S. Mrenna and P. Z. Skands, Comput. Phys. Commun. 178 (2008) 852.

[29] S. Carrazza, S. Forte and R. Rojo, arXiv: 1311.5887 [hep-ph].

[30] ATLAS Collaboration, ATL-PHYS-PUB-2014-021, url: http://cds.cern.ch/record/1966419.

[31] S. Alioli et al., JHEP 06 (2010) 043.

[32] E. Bagnaschi et al., JHEP 02 (2012) 088.

[33] T. Gleisberg et al., JHEP 02 (2009) 007.

[34] S. Schumann and F. Krauss, JHEP 03 (2008) 038.

[35] S. Höche et al., JHEP 05 (2009) 053.

[36] ATLAS Collaboration, JHEP 01 (2013) 086.

[37] ATLAS Collaboration, Phys. Rev. D 85 (2012) 012003.

[38] T. Binoth et al., Eur. Phys. J. C 16 (2000) 311.

[39] ATLAS Collaboration, ATLAS-CONF-2016-059, url: http://cds.cern.ch/record/2206154. 